\begin{tabular}{ccc}
\hline & International Journal of Engineering \& Technology, 7 (2.33)(2018) 628-631 \\
SPC & Website: www.sciencepubco.com/index.php/IJET \\
Research paper & Technology \\
\hline
\end{tabular}

\title{
Analytical modeling of the human cardiovascular system with real-time implementation using NI ELVIS-II
}

\author{
Sisir Chettri ${ }^{1}{ }^{*}$, Akash Kumar Bhoi ${ }^{1}$, Gyoo-Soo Chae ${ }^{2}$, Nilas Gurung ${ }^{1}$, Ashis Sharma ${ }^{3}$ \\ ${ }^{1}$ Department of Electrical \& Electronics Engineering, Sikkim Manipal Institute of Technology (SMIT), Sikkim Manipal University, \\ India \\ ${ }^{2}$ Division of Information and Communication, Baekseok University, Cheonan, South Korea \\ ${ }^{3}$ Sikkim Manipal Institute of Technology (SMIT), Sikkim Manipal University, India \\ *Corresponding author E-mail: akash730@gmail.com
}

\begin{abstract}
Cardiovascular System, which consists of the heart, the systematic circulation and the pulmonary circulation is said to be the transport system for the human body. Modeling of cardiovascular system has become important for clinical researchers and for deeper understanding of blood circulation in the human body. This paper uses the lumped method which is also known as an electrical analogy for modeling and simulation of human cardiovascular system. A simplified complete lumped parameter model of the Human Cardiovascular System has been developed with real time implementation focusing mainly on blood flows. A resistor, an inductor and a capacitor are used to model every blood vessel, ventricles, atrium and set of all veins and capillaries. A pulse generating circuit is also modeled which acts as a power supply for the heart that controls the contraction of heart muscles.
\end{abstract}

Keywords: Lumped Parameter; Electrical Analogy; Cardiovascular System; NI ELVIS II; Sinoatrial Node Pulse Generator; Blood Circulation

\section{Introduction}

The main task of the cardiovascular system is supplying the organs with blood. Human cardiovascular system is composed of the heart, the systemic circulation and the pulmonary circulation. Oxygenated blood is circulated to various organs by the systematic circulation, whereas the pulmonary circulation carries blood between the heart and the lungs. Human cardiovascular diseases are considered the most disastrous health problems as it causes most of death in industrialized nations. For better understanding of the cardiovascular system functionality mathematical modeling is one of the important, most useful, and accurate method. There are different ways which can be used for analyzing the cardiovascular system and effects of diseases on it which includes the lumped model, one, two or threedimensional modeling and experimental methods [1]. A physical model can be used to understand the fundamental concepts more deeply. There are different methods that vary in complexity and purpose. In this paper an attempt has been made to represent the human cardiovascular system by an equivalent electronic circuit The equivalent electrical circuit consists of a resistor, a capacitor and an inductor representing the arteries, arterioles, capillary beds, and veins in the physiological context. The heart acts as a power supply for the whole cardiovascular system. The pumping action of the heart circulates nutrient blood to different organs, tissues and the muscles [2]. In this paper different types of pulse generation circuits were also analyzed which can be used to generate periodic electrical impulses that control the contraction of heart muscles.

\section{Modeling principles}

In this project, the earlier existing model of the human cardiovascular system using the lumped method was studied. The simplified model helps to understand the functionality in a better way [3]. New equivalent electrical circuit with certain modifications on the existing model of the cardiovascular system has been designed. It was found that the ventricles are the power supply of heart. So, the supply for the model is given to left ventricles and right ventricles. Also, left ventricles have the highest pressure [4]. So, supply with different output voltage magnitude is designed and the one with higher output is fed to the left ventricles. In this electrical model of the cardiovascular system, every component of the cardiovascular system is represented by a block consisting of a resistor, an inducer, a capacitor and a diode. Valves in the heart prevent backflow of the blood as a result, it can be modeled using a diode which does a similar work in the electrical system. Every parameter of electrical model is related to the cardiovascular system. As shown in fig. 1 and fig. 2 the elements of the cardiovascular system are represented with equivalent electrical circuit where the voltage is equal to blood pressure and charge is equal to the volume.

The elements of the cardiovascular system are related to elements of the equivalent electrical circuit as,

$0.01 \mathrm{ml} / \mathrm{Pa}=[1] \mu \mathrm{F}$ (compliance - capacitance), $1 \mathrm{~Pa} . \mathrm{s}^{2} / \mathrm{ml}=1 \mu \mathrm{H}$ (inertia -inductor), $1 \mathrm{~Pa} . \mathrm{s} / \mathrm{ml}=1 \mathrm{k} \Omega$ (resistance - resistance), $1 \mathrm{mmHg}=1$ volt (pressure - voltage), $133416 \mathrm{ml}=1 \mathrm{~A}($ volume charge) [5]. 


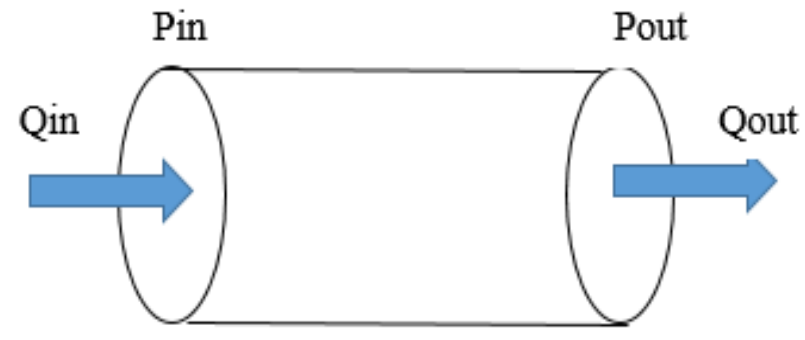

Blood in

Fig. 1: Blood Vessel.

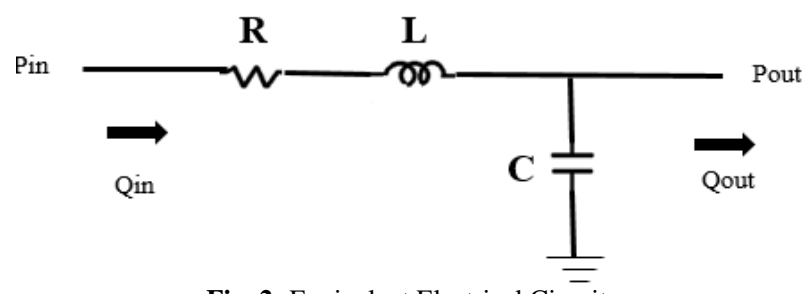

Fig. 2: Equivalent Electrical Circuit.

\section{Circuit description}

The equivalent circuit can be subdivided into different parts as the sinoatrial node pulse generator, the heart, the pulmonary circuit and the systematic circuit. In biological field the sinoatrial node in the human body produces electrical impulses that generate the signal which is necessary for contraction of the heart muscles. In that context the name sinoatrial node pulse generator is used here, which provide power to the heart muscles for the necessary contractions required to perform the blood circulation. Firstly, the modeling was done for both sinoatrial node pulse generator and the cardiovascular system. After that the simulation was carried out in multisim software followed by the hardware implementation of the whole cardiovascular system.

\section{Simulation}

Simulation for the sinoatrial node pulse generator and the cardiovascular system was implemented using the multi-user software. The results thus obtained were analyzed and verified with the standard results.

\subsection{Sinoatrial node pulse generator}

Fig. 3 shows the electrical circuit of the sinoatrial node pulse generator. A train of pulse is applied to the base of bipolar junction transistor. The circuit produces a constant train of pulses with a certain amount of droop which can be used as a power supply to the heart, which is necessary for contraction of heart muscles.

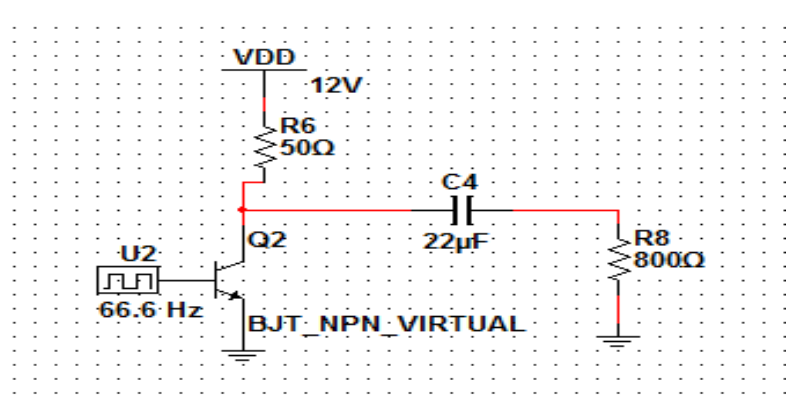

Fig. 3: Sinoatrial Pulse Generation Circuit.

\subsection{Cardiovascular system}

Work on modeling of human cardiovascular system has been carried out before by another research team. The existing model with certain modifications has been presented in this paper. Fig.4 shows the electrical circuit representation of the cardiovascular system. The value of flow resistance (R), blood inertia (L) and compliance (C) are calculated from the following formulas (eq. 1, 2 and 3) whose values depend on blood vessel properties (diameter, length, wall thickness, viscosity, etc.)

The flow resistance is given as (eq. (1)):

$\mathrm{R}=\frac{8 \mu \mathrm{l}}{\pi \mathrm{r} 4}$

Where, $\mu$ is blood viscosity, 1 is the length and $r$ are the radius of each segment.

$$
\mathrm{C}=\frac{31 \pi \mathrm{r}^{3}}{2 \mathrm{Eh}}
$$

Where, $r, E, h$ is radius, elasticity modulus and thickness of arteries respectively.

$$
\mathrm{L}=\frac{91 \rho}{4 \mathrm{~A}}
$$

Where, $\mathrm{L}$ is blood inertia and $\rho$ is blood density and A is cross sectional area of each artery.

Length and diameter of left ventricles are $0.5523 \mathrm{~cm}$ and $0.63 \mathrm{~cm}$ [3]. So, R and $\mathrm{L}$ can be calculated using the formula which gives $\mathrm{R}=0.50050 \mathrm{kohm}$ and $\mathrm{L}=0.10036 \mu \mathrm{h}$. The structure of left heart is same as that of right heart as a result the component valve of right heart is same as that of the left heart. The only difference is that left heart has higher pressure. Most of the values for the electrical components have been referred to the earlier existing models [6]. Advancement in solid-state devices makes it possible to study the behavior of fundamental components and monitor the parameters in more details [7].

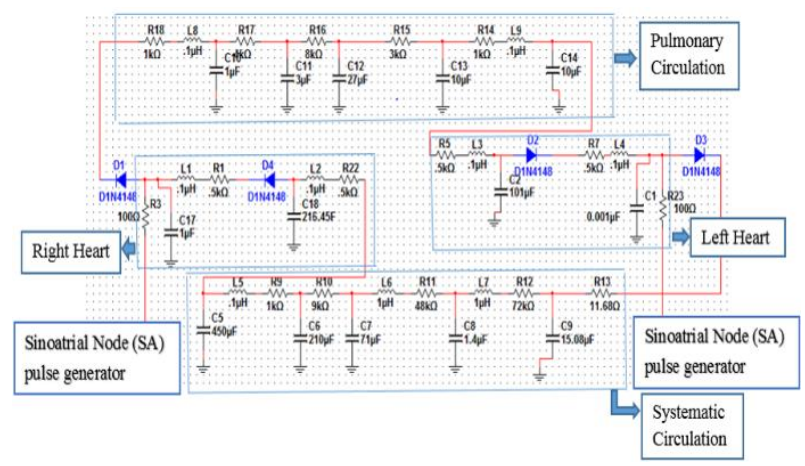

Fig. 4: Electrical Circuit Representation of the Cardiovascular System.

\section{Hardware implementation}

The simulated circuits for both sinoatrial node pulse generator and cardiovascular system were implemented in hardware. The pulse generator circuit was designed in breadboard and cardiovascular system in NI ELVIS II.

\subsection{Sinoatrial node pulse generator circuit}

Two sinoatrial node pulse generator circuit were designed and fed to the heart as a power supplier which is required for contraction of heart muscles. The one with higher voltage magnitude is fed to the left ventricles and the one with lower voltage magnitude to right ventricles. Fig. 5 shows hardware implementation of Sinoatrial node pulse generator. 


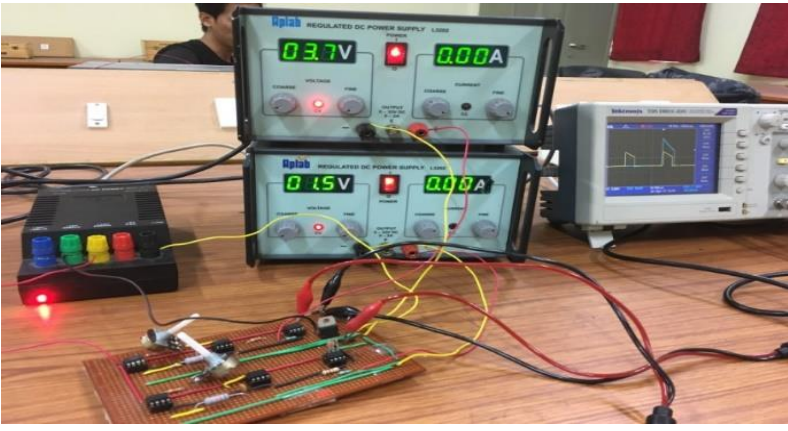

Fig. 5: Sinoatrial Node Pulse Generator Circuit.

\subsection{Hardware implementation of cardiovascular system in NI ELVIS II}

The simulated result which was obtained in multisim was implemented in the NI ELVIS kit and the result was verified. Fig.6 shows the hardware implementation of equivalent electrical circuit of cardiovascular system.

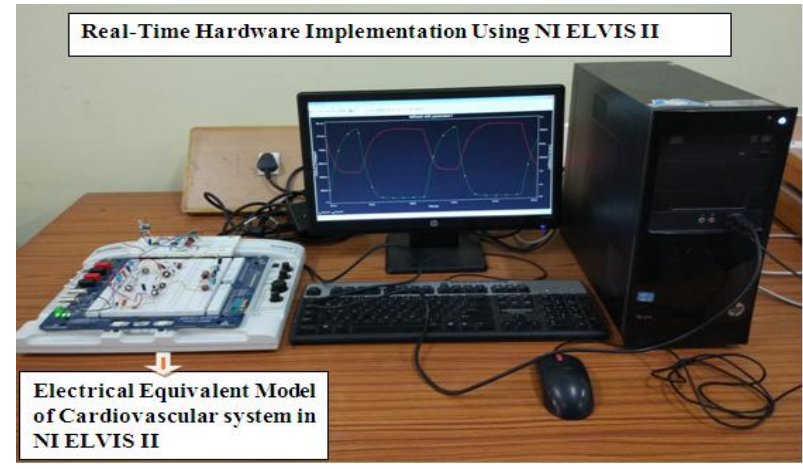

Fig. 6: Hardware Implementation in NI ELVIS II.

\section{Results}

The simulated and hardware results of sinoatrial node pulse generator circuit were analyzed and were fed to the ventricle of the heart Fig. 7 and fig. 8 shows the simulated and hardware output of sinoatrial node pulse generator respectively.

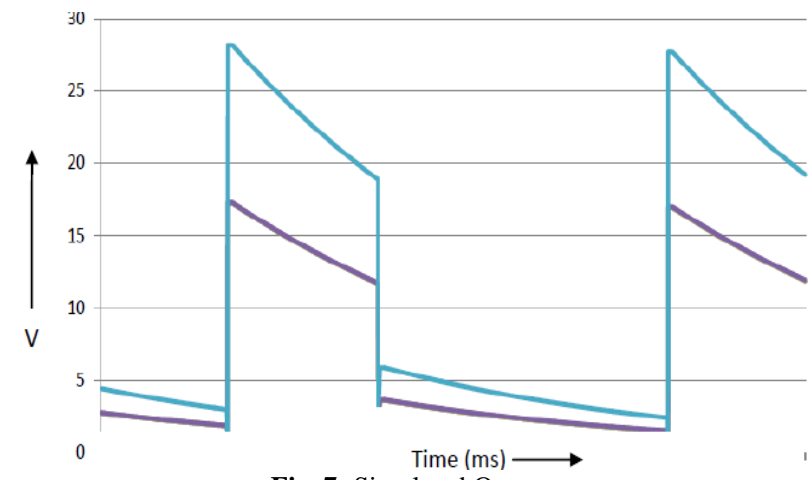

Fig. 7: Simulated Output.

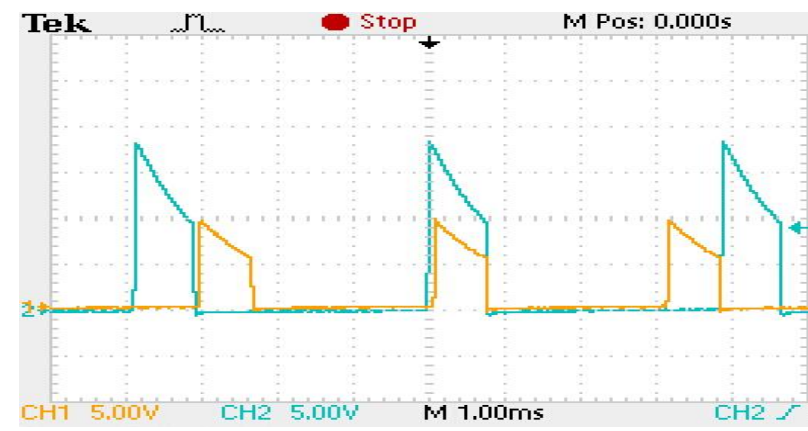

Fig. 8: Hardware Output.
The area covered by the output pulse gives the energy. This pulse is fed to the heart for necessary contraction of heart muscles in the equivalent electrical circuit of cardiovascular system.

Fig.9 shows the output pressure waveform of right ventricles and fig. 10 shows the output pressure waveform of left ventricles. The pressure of right ventricles varies between (27-10) $\mathrm{V}$ and pressure of left ventricles varies between (110-20) V which is in complete accordance with the physiological data.

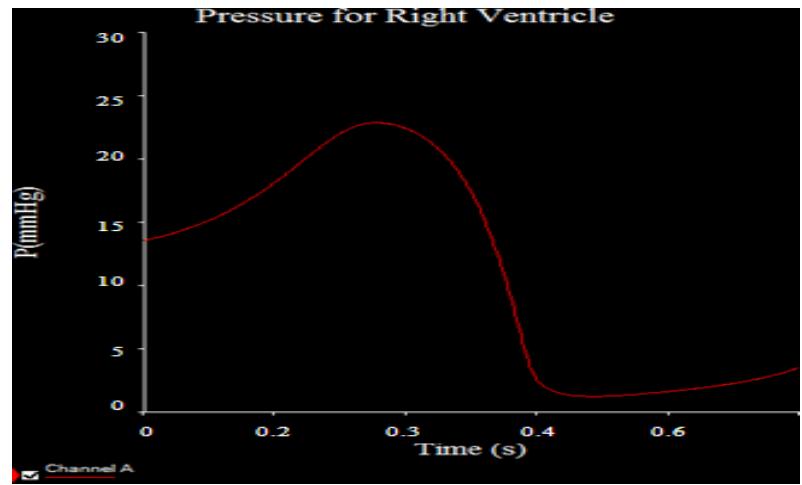

Fig. 9: Output Pressure Waveform of Right Ventricles.

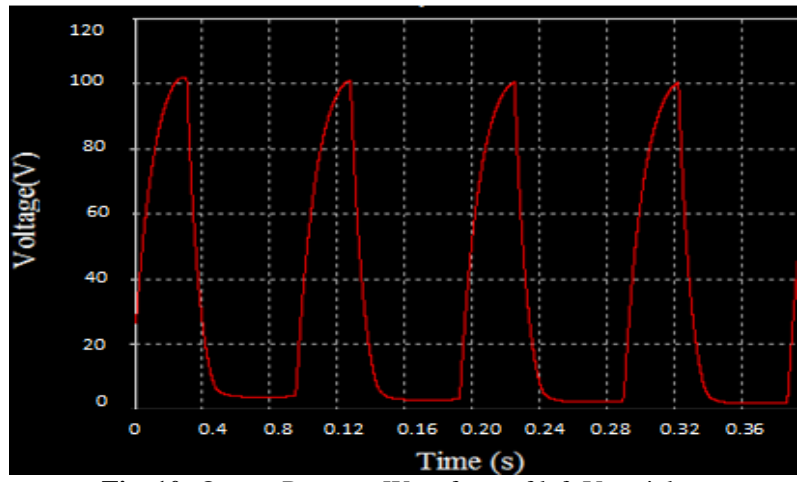

Fig. 10: Output Pressure Waveform of left Ventricles.

\section{Conclusion}

In this paper an equivalent model of human cardiovascular system has been modified and implemented. Lumped method was used for the modeling purpose. Modeling of the cardiovascular system is useful for investigation of the cardiovascular system and various types of abnormalities associated with it. The heart was fed from the pulse generating circuit which acts as a power supplier that generates an electrical signal necessary for contraction of heart muscles. For these reason two types of pulse generating circuit has been modeled and simulated. Finally, the pulse generating circuit was fed into the electrical equivalent cardiovascular system to obtain pressure graphs of the left and right ventricles. By varying the electrical parameters as per the modelling principles, different abnormalities can be studied with the change in the output waveform in a noninvasive and more accurate method. This paper model of equivalent electrical cardiovascular system has been successfully implemented. The same concept can be implemented at a higher level for better understanding of cardiovascular system.

\section{References}

[1] O. Ghasemalizadeh, M. R. Mirzaee, B. Firoozabadi (2008), Modeling The human cardiovascular system and peristaltic motion of descending arteries using the lumped method. The Internet Journal of Bioengineering, Vol.3 number 1 .

[2] Hassanain Ali Lefta Mossa: Engineering Modeling of Human Cardiovascular System (2008). The first Regional Conference of Eng. Sci. NUCEJ Spatial, vol.11, No.2, pp.307-314.

[3] F.E Cellier, E. kofman (2006), continuous system simulation. Springer-science, New York. 
[4] Vera Höfflin, Amiliana M Soesanto1, Hananto Andriantoro, Eko Supriyanto, Jens Haueisen (2015), Modelling and Simulation of Blood Circulation towards Mitral Valve Replacement Risk Calculation. Fourth International Conference on Instrumentation, Communications, information Technology, and Biomedical Engineering (ICICIBME) Bandung.

[5] Canek Phillips (2015), a Simple Lumped Parameter Model of the Cardiovascular System. Thesis for the Degree of Master of Science colorado State University Fort Collins, Colorado.

[6] Wu, X.M (1994), Modelling and simulation of cardiovascular circulation system, Status and prospect. Science- paper Online, vol.2, pp.112-116.

[7] Milišić, V. and Quarteroni (2004), Analysis of lumped parameter models for blood flow simulations and their relationship with 1D models. Mathematical Modeling and Numerical Analysis, pp. 613632. 